\title{
Dynamic Threshold Energy Detection Technique for Cognitive Radio
}

\author{
Neelu \\ Electronics and Communication Engineering, \\ IFTM University, \\ Moradabad, India
}

\author{
Arun Kumar \\ Electronics and Communication Engineering, \\ IFTM University, \\ Moradabad, India
}

\begin{abstract}
Spectrum sensing is the main feature of cognitive radio technology. Spectrum sensing gives an idea of detecting the presence of the primary users in a licensed spectrum. The dynamic threshold energy detection is proposed in this paper for the spectrum sensing of Cognitive Radio. The proposed dynamic energy scheme depends on the current state of the primary user. Depending on this, dynamic thresholds are evaluated considering the effect of noise uncertainty. The thresholds evaluated are used to increase the value of $P_{d}$ and decrease the value of $P_{f a}$. The performance of dynamic energy detection technique is optimized against the values of parameters. It is observed that the proposed dynamic energy detection (DED) scheme is better than the classical energy detection (CED) scheme.
\end{abstract}

\section{Keywords}

Spectrum sensing, Energy detection, Probability of detection $\left(P_{d}\right)$, Probability of false alarm $\left(P_{f a}\right)$

\section{INTRODUCTION}

Cognitive radio (CR) [1] technique has been initiated to exploit the spectrum holes when the spectrum is under utilization. It allows the secondary users called CR user to access the spectrum along with primary users (PU) in opportunistic manner. A CR basically senses the spectrum, understands its operating environment, identify the available temporary spectrum, adapt spectrum for transmission and learn its behavior.

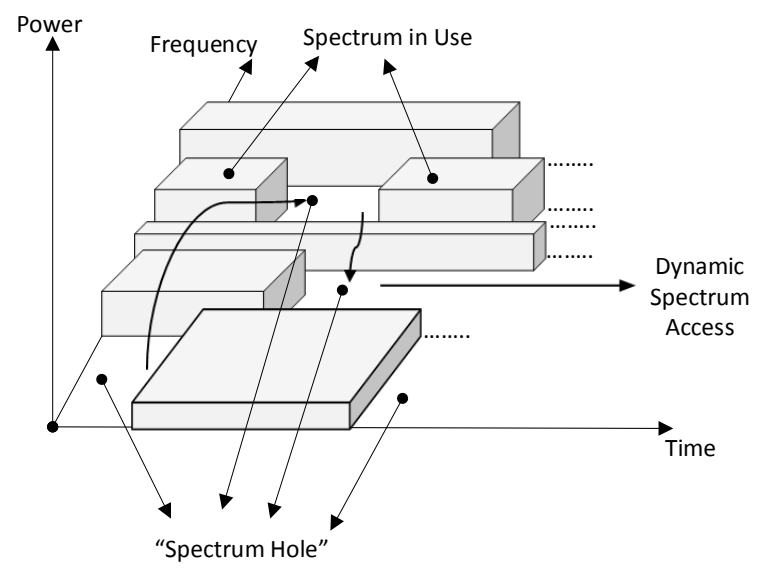

Fig 1: Concept of frequency spectrum hole [1]

A frequency range hole (sometimes known to be White Space) shown in fig.1, is a range of frequencies which is designated to the PU, but the frequency range remains unutilized by the user at any time and geographic area. The concept of frequency range hole can be revised as a probability in transmission in space of Electromagnetic (EM) spectrum. EM spectrum area is not a practical space which is owned by transmitted signals having dimension of location, frequency, arrival angle, power, time and many more as possible. The radio which is built on CR has abilities of sensing and understanding the frequency band, recognize the frequency range holes in electromagnetic spectrum, in making decision of how the user can access the electromagnetic range and to adapt it transmission and reception parameters accordingly. Therefore, the cognitive radio that utilize this strong electromagnetic range access involves the capability to significantly enhance the frequency band efficiency utilization that results in easier and flexibility of accessing the spectrum for present and upcoming services of wireless communication.

The concept of Cognitive Radio was given by Gerald Maguire and Joseph Mitola [2]; they expressed the expansion of Software defined radio that improves the performance of wireless communication.

In this paper a dynamic energy scheme that depends on the current state of the primary user is developed. Depending on this, dynamic thresholds are evaluated and considering the effect of noise uncertainty. The thresholds evaluated are used to increase the value of $P_{d}$ and decrease the value of $P_{f a}$. The performance of dynamic energy detection technique is optimized against different values of parameters.

\section{SYSTEM MODEL}

Energy detection is the most common technique used in the detection of primary transmitter. In this technique, the detector performs non-coherent detection and therefore does not require any prior knowledge of the primary transmitter [35]. Fig. 2, shows the block diagram of the energy detection. The signal $\mathrm{Y}(\mathrm{f})$ is obtained by passing the received signal $\mathrm{y}(\mathrm{t})$ through band pass filter and Fast Fourier Transform. The energy gain of the signal is then calculated by integrating and summing the information $\mathrm{Y}(\mathrm{f})$ over detection interval of time. The primary user is then encountered by correlating energy of received signal with the range of threshold. The threshold decision confides upon the level needed for fake alarm and is much inclined to the uncertainty in noise signal. This technique cannot differentiate between noise and primary signal and therefore has very low attainment under less SNR. Also this technique is unsuitable for the detection of spread spectrum signals due to complex signal processing algorithms used for the detection. 


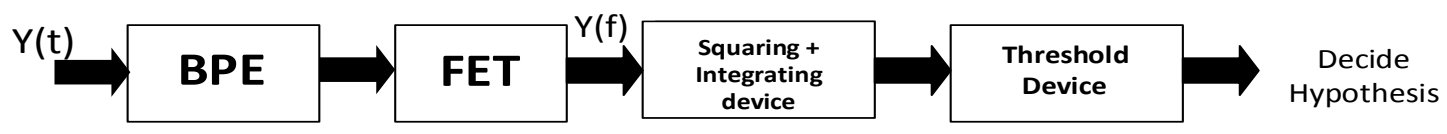

Fig 2: Block diagram of energy detection

\subsection{Spectrum Sensing Problem Formulation}

With the help of two hypotheses of binary hypothesis problem of testing, we can formulate spectrum sensing problem as described below:

$$
\begin{aligned}
& H_{0} \text { denotes }: y(n)=w(n) \rightarrow(P U \text { unavailable }) \\
& H_{1} \text { denotes }: y(n)=x(n)+w(n) \rightarrow(P U \text { available })
\end{aligned}
$$

Here, $y(n)$ is the signal received, by primary user, $n=1,2$, $3 \ldots \mathrm{N}$ where $\mathrm{N}$ is complete primary legal user's observing period length. $x(n)$ represents the sample of primary legal user signal while $w(n)$ represents the sample of AWGN (Additive White Gaussian Noise) having variance $\sigma_{n}^{2}[6]$. Hypotheses $H_{0}$ say about the absence of primary user and Hypotheses $H_{1}$ say about the presence of primary user. The samples of noise are related to variance in way as mentioned:

$$
w(n) \in N\left(0, \sigma_{n}^{2}\right)
$$

When the spectrum sensing fails then the resulting probability is known as probability of false alarm and probability of missed detection. In case probability of missed detection, when primary user is available in spectrum band then the technique of spectrum sensing selects hypotheses $H_{0}$. In case probability of false alarm, when the frequency band is sitting idle then the technique now selects hypotheses $H_{1}$. In probability of missed detection, there is a consequence of resulting interference to the primary user. While in probability of false alarm it results in missing the opportunities and due to which the utilization of spectrum in an effective and efficient manner decreases. Depending on these two definitions of probability, it can be concluded that there are two types of probabilities namely, probability of detection and probability of false alarm [7]. For the optimal performance of cognitive radio it is expected that probability of detection should be maximized and probability of false alarm should be minimized.

The probability of detection, $P_{d}=P_{r}\left(H_{1} / H_{1}\right)$ and

Probability of false alarm, $P_{f a}=P_{r}\left(H_{1} / H_{0}\right)$.

\section{COMPARISON ANALYSIS OF CED AND DED}

\subsection{Fixed Threshold Classical Energy Detector}

Usually cognitive radio do not contain information about the primary user, in this case energy detection plays a very vital role. As energy detection can analyze the availability of primary user when it is not having any prior information about it. Also, the implementation and computation is very effective and easy in this detection technique.

In energy detection technique considering fixed threshold, it analyzes the availability of primary user in the spectrum range. If the energy which is received from primary user comes out to be greater than the fixed threshold that was predetermined, the spectrum frequency band is considered to be busy and declares Hypotheses $H_{1}$. And if the energy received comes out to be less than the predetermined fixed threshold value, the spectrum can be declared as idle and hypotheses $H_{0}$ is considered in this case [8].

The statistic to be tested is given as:

$$
D(y)=\sum_{n=0}^{N} y(n)^{2}
$$

Here, $y(n)$ denotes the received energy of primary signal, $D(y)$ is the decision variable. The statistic to be tested follows central distribution chi-square beneath hypotheses $H_{0}$ and non-central distribution beneath hypotheses $H_{1}$. While considering the region having low signal-to-noise-ratio, the sample number needed to gain certain performance in probability of false alarm and probability of detection should always be more than a single sample. The Central Limit Theorem and Gaussian distribution is used to analyze the statistic to be tested, that is described below [9];

$$
D(y)=\left\{\begin{array}{c}
N\left(N \sigma_{n}^{2}, 2 N \sigma_{n}^{4}\right) H_{0} \\
N\left(N\left(P+\sigma_{n}^{2}\right), 2 N\left(P+\sigma_{n}^{2}\right)^{2}\right) H_{1}
\end{array}\right.
$$

$\mathrm{P}$ is the average signal power of Primary user. The probability of false alarm and probability of detection is described as below if the Additive White Gaussian Noise (AWGN) is considered:

$$
\begin{gathered}
P_{d}=P_{r}\left(D(y)>\gamma \mid H_{1}\right)=Q\left(\frac{\gamma-N\left(P+\sigma_{n}^{2}\right)}{\sqrt{2 N}\left(P+\sigma_{n}^{2}\right)}\right) \\
P_{f a}=P_{r}\left(D(y)>\gamma \mid H_{0}\right)=Q\left(\frac{\gamma-N \sigma_{n}^{2}}{\sqrt{2 N} \sigma_{n}^{2}}\right)
\end{gathered}
$$

$P_{d}$ denotes the probability of detection and $P_{f a}$ denotes the probability of false alarm. Q(.) is the standard Gaussian CDF (Cumulative distribution function) and $\gamma$ is the predetermined fixed threshold.

The desired predetermined fixed threshold can be calculated by [10]:

$$
\gamma=\sigma_{n}^{2}\left[Q^{-1}\left(P_{f a}\right) \sqrt{2 N}+N\right]
$$

From the value of threshold evaluated above, it can be seen that the threshold not only depends on probability of false alarm but also on the noise variance. As the signal changes, noise variance also changes but due to the fixed value of threshold, the performance of energy detector does not come out to be optimum. Also, a small change in noise variance can highly provide a harmful impact on the value of threshold. So, there is a need to change the value of threshold considering the effect of noise uncertainty. Here, comes the effect for the dynamic threshold energy detection. 


\subsection{Dynamic Threshold Energy Detection Technique}

In this section, a new technique is proposed for energy detection based on dynamic threshold considering the effect of noise uncertainty. This consideration of noise uncertainty factor makes the cognitive radio perform in a better way than the predetermined threshold fixed energy detector. In this detection technique, it is required to calculate two values of threshold so as to maximize the probability of detection and minimize the probability of false alarm. From the values of probability of detection and probability of false alarm, it can be seen that, one can estimate the availability of legal user in the present stage; one can increase the probability of detection and decrease the probability of false alarm. This can be achieved by decreasing and increasing the already used levels of threshold. Using all these artifacts a new dynamic energy detection scheme is proposed by predicting the primary user. Through this detection mechanism, the average energy received by the legal primary user is evaluated and compare this average energy with the threshold value. The energy received is considered during the $\mathrm{L}$ consecutive $\mathrm{N}$ observation periods.

The average energy received by the primary legal user is defined as below [11-12]:

$$
D_{a v}=\frac{1}{L} \sum_{k=1}^{L} D(y)_{k}
$$

$D(y)_{k}$ is the energy received. Noise uncertainty factor can be calculated by dividing the maximum value of noise variance by the average value of all the noise variances.

The noise uncertainty factor is given as below:

$$
\rho=\frac{\max _{1 \leq i \leq L}\left(\sigma_{n_{i}}^{2}\right)}{\frac{1}{L} \sum_{i=1}^{L} \sigma_{n_{i}}^{2}}
$$

After the estimation of average received energy and noise uncertainty factor, the average energy with the predetermined threshold $(\gamma)$ is compared.

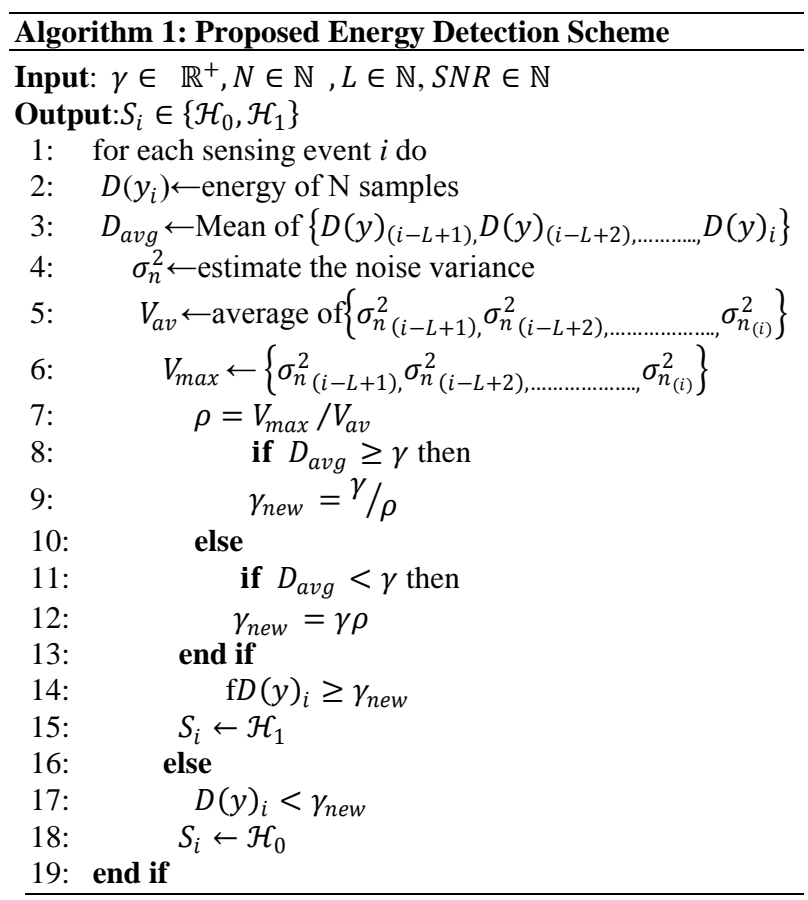

The two different cases for evaluating the new thresholds taking into account the effect of noise uncertainty is also considered.

Case 1.If $D_{a v} \geq \gamma$, the estimation of the presence of primary user (line 8 in algorithm 1), and new threshold is calculated by considering the effect of noise uncertainty i.e.

$$
\gamma_{\text {new }}=\gamma / \rho
$$

Case 2.If $D_{a v}<\gamma$, the estimation about the absence of primary user (line 11 in algorithm 1), and the new threshold is evaluated by including the effect of noise uncertainty as,

$$
\gamma_{\text {new }}=\gamma \rho
$$

Now, dynamic nature of this proposed algorithm will highly undergo the effect of noise uncertainty in the evaluation of primary user's state. Hence, dynamic threshold scheme provides better result as compared to conventional energy detection scheme. The dynamic threshold scheme involves the concept of fixed threshold without considering the effect of noise uncertainty.

\subsubsection{Theoretical Analysis of the New Proposed Scheme}

Depending upon what the algorithm estimate about primary user and what the actual status is about, the theoretical study of our technique is divided into four cases:

Case1. True prediction:

PU is actually present, algorithm also declared hypotheses $H_{1} \cdot \gamma_{\text {new }}=\gamma / \rho$ the new threshold is proposed that will increase the value of probability of detection.

Case2. False prediction:

PU is actually absent, algorithm declared hypotheses $H_{1}$. $\gamma_{\text {new }}=\gamma / \rho$ the new threshold is proposed that will increase the value of probability of false alarm.

Case3. True prediction:

PU is actually absent; algorithm also declared hypotheses $H_{0} \gamma_{\text {new }}=\gamma \rho$ the new threshold is proposed which will decrease the probability of false alarm.

Case4. False prediction:

PU is actually present, algorithm declared hypotheses $H_{0 .} \gamma_{\text {new }}=\gamma \rho$ the new threshold is proposed and it will decrease the value of probability of detection.

Table1. Theoretical analysis of proposed energy detection scheme

\begin{tabular}{|l|c|c|c|c|}
\hline Case & $\mathbf{1}$ & $\mathbf{2}$ & $\mathbf{3}$ & $\mathbf{4}$ \\
\hline Predicted State & $H_{1}$ & $H_{1}$ & $H_{0}$ & $H_{0}$ \\
\hline Actual State & $H_{1}$ & $H_{0}$ & $H_{0}$ & $H_{1}$ \\
\hline New threshold & $\gamma / \rho$ & $\gamma / \rho$ & $\gamma \rho$ & $\gamma \rho$ \\
\hline Result & $P_{d} \uparrow$ & $P_{f a} \uparrow$ & $P_{f a} \downarrow$ & $P_{d} \downarrow$ \\
\hline
\end{tabular}

\section{RESULT AND DISCUSSIONS}

In this section, different parameters are used to evaluate the performance of CED and DED through MATLAB. Figures 3 to 6 shows the receiver operating curves $\left(P_{d} V s P_{f a}\right)$ that are drawn for CED and DED technique. To know the performance of these techniques different values of $\mathrm{L}, \mathrm{N}$ and SNR are chosen. 


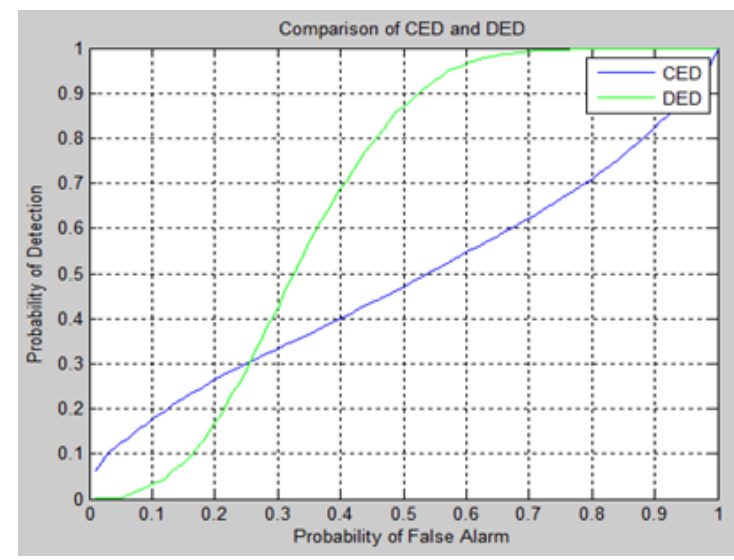

Fig 3: Comparison of CED and DED $(L=50, N=1000$, SNR=-20db)

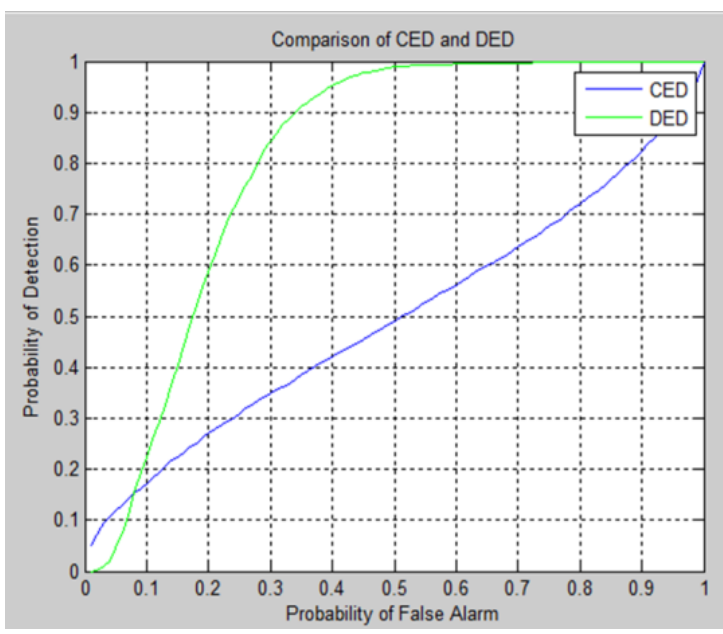

Fig 4: Comparison of CED and DED ( $L=200$, $\mathrm{N}=1000, \mathrm{SNR}=-20 \mathrm{db}$ )

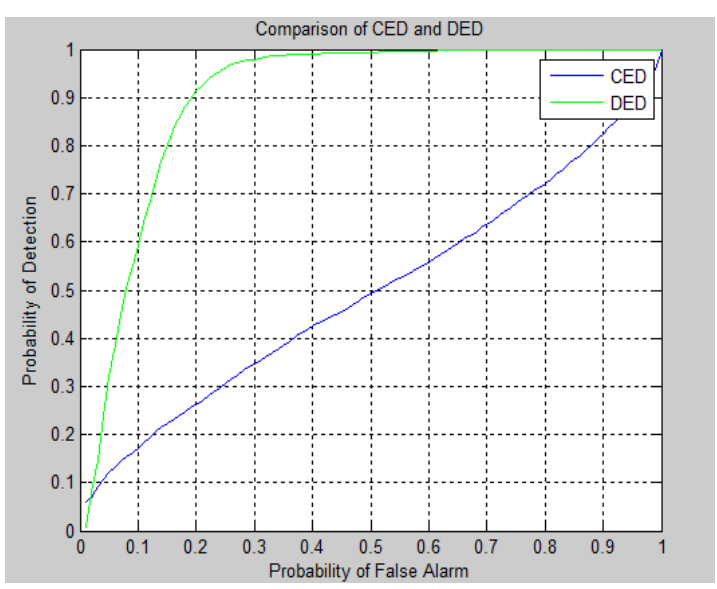

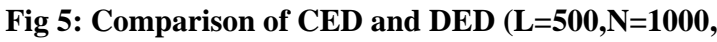
$\mathrm{SNR}=-20 \mathrm{db}$ )

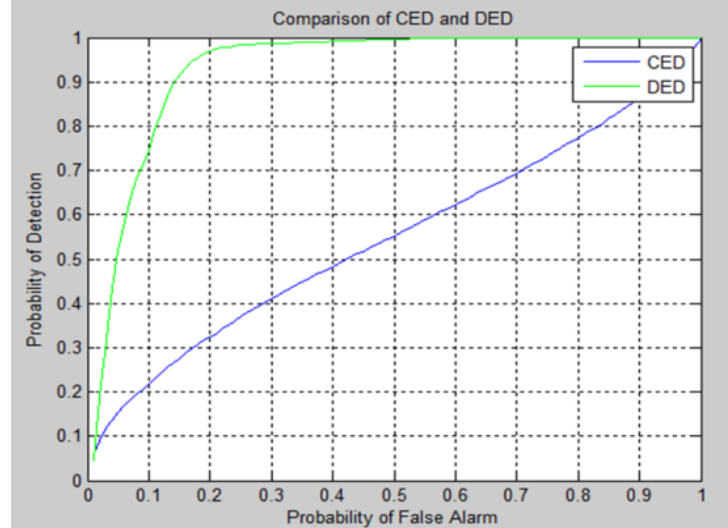

Fig 6: Comparison of CED and DED $(L=500, N=1000$, SNR=-10db)

In the above graphs, Classical Energy Detector (CED) and Dynamic Energy Detector (DED) have compared. It is clear from the above figures that DED increase in probability of detection with minimum probability of false alarm.

\section{CONCLUSION}

The dynamic threshold energy detection is proposed in this paper for the spectrum sensing of Cognitive Radio. The proposed dynamic energy scheme depends on the current state of the primary user. Depending on this, dynamic thresholds are evaluated considering the effect of Noise Uncertainty. The thresholds evaluated are used to increase the value of $P_{d}$ and decrease the value of $P_{f a}$. The performance of dynamic energy detection technique is optimized against the different values of parameters. It is clear from the figures proposed dynamic energy detection (DED) scheme is better than the classical energy detection (CED) scheme.

\section{REFERENCES}

[1] S.Haykin, "Cognitive radio: brain empowered wireless communications," IEEE Journal on Selected Areas in Communications, vol.23, pp. 201-220, Feb. 2005.

[2] Mitola III, Joseph, and Gerald Q. Maguire Jr. "Cognitive radio: making software radios more personal. " IEEE Personal Communications., 1999,6 (4),13-18.

[3] T. Yucek and H, Arslan, "A survey of spectrum sensing algorithms for cognitive radio applications," IEEE Communications Surveys and Tutorials, vol. 11, pp. 116130, Jan. 2009.

[4] G. Yu, C. and W. Xi, "A novel energy detection scheme based on dynamic threshold in Cognitive radio systems," Journal of Computational Information Systems, vol. 8, pp. 2245-2252, Mar. 2012

[5] X. Hue, X. Xie, T.Song and W. Lei, "An algorithm for energy detection based on noise variance estimation under Noise Uncertainty," IEEE International Conference on Communication and Technology, pp. 1-5, Nov, 2012

[6] Y. Zeng and Y.-C. Liang, "Maximum-minimum eigenvalue detection for cognitive radio," in Proc. IEEE International Symposium on Personal, Indoor and Mobile Radio Communication, (PIMRC'07), pp. 1-5, 2007.

[7] F. Digham, M. Alouini, and M. Simon, "On the energy detection of unknown signals over fading channels," 
IEEE Transactions on Communications, vol. 55, pp. 2124, Jan. 2007.

[8] J. Song, Z. Feng, P. Zhang, and Z. Liu, "Spectrum sensing in cognitive radios based on enhanced energy detector," IET Communications, vol. 6, pp. 805-809, May 2012.

[9] Y. Zeng, Y.-C. Liang, A. Hoang, and C. Peh, "Reliability of spectrum sensing under noise and interference uncertainty," in Proc. IEEE International Conference on Communications Workshops, (ICC'09), pp. 1-5, 2009.

[10] Akyildiz, I.F., Lee, W.-Y., Vuran, M.C., Mohanty, S.: 'A survey on spectrum management in cognitive radio networks', IEEE Commun. Mag., 46, (4), pp. 40-48, 2008 .
[11] Yücek, T., Arslan, H.: 'A survey of spectrum sensing algorithms for cognitive radio applications', IEEE Commun. Surv. Tutor (First Quarter), 11, (1), pp. 116130, 2009.

[12] Ariananda, D.D., Lakshmanan, M.K., Nikookar, H.: 'A survey on spectrum sensing techniques for cognitive radio'. Proc. Second Int. Workshop on Cognitive Radio and Advanced Spectrum Management (CogART 2009), pp. 74-79, May 2009.

[13] Zeng, Y., Liang, Y.-C.: 'Spectrum-sensing algorithms for cognitive radio based on statistical covariances', IEEE Trans. Veh. Technol, 58, (4), pp. 1804-1815, 2009. 